\title{
Assistant MEEGA+: Uma ferramenta de apoio para avaliação de jogos educacionais usando modelo MEEGA+
}

\section{Ronan Soares $^{1,2}$, Giani Petri ${ }^{3,4}$, Christiane Gresse von Wangenheim ${ }^{3}$, Tayana Conte $^{5}$, Anna Beatriz Marques ${ }^{6}$}

\author{
${ }^{1}$ UNINORTE Laureate Universities - Manaus - AM - Brasil \\ ${ }^{2}$ Fundação Paulo Feitoza (FPF TECH) - Manaus - AM - Brasil
}

${ }^{3}$ Grupo de Qualidade de Software (GQS) - Programa de Pós-Graduação em Ciência da Computação (PPGCC) - Departamento de Informática e Estatística (INE) Universidade Federal de Santa Catarina (UFSC) - Florianópolis - SC - Brasil

${ }^{4}$ Universidade Federal de Santa Maria (UFSM) - Santa Maria - RS - Brasil

${ }^{5}$ Grupo de Pesquisa em Usabilidade e Engenharia de Software (USES) - Instituto de Computação - Universidade Federal do Amazonas (UFAM) - Manaus - AM - Brasil

$$
\begin{gathered}
{ }^{6} \text { Universidade Federal do Ceará (UFC) - Russas - CE - Brasil } \\
\text { ronan.ripasy@gmail.com, gpetri@inf.ufsm.br, c.wangenheim@ufsc.br, } \\
\text { tayana@icomp.ufam.edu.br, beatriz.marques@ufc.br }
\end{gathered}
$$

Abstract. Educational games are an innovative approach for teaching. It is necessary to evaluate games to obtain evidence about the benefits of adopting them. MEEGA+ is a model for evaluating games used for teaching computer science and analyses the experience and perception about learning from the point of view of students and instructors. Although providing templates and predefined spreadsheets, there is not a comprehensive tool providing support for the complete evaluation process. This paper presents the Assistant $M E E G A+$, a web support tool for MEEGA+. An evaluation of the Assistant $M E E G A+$ by instructors and professionals interested in developing and evaluating educational games indicated positive results for the usefulness, ease of use and intention to use the tool. The results encourage the educational games evaluation and the Assistant MEEGA+ adoption.

Resumo. Jogos educacionais são uma estratégia inovadora para o ensino. Todavia é necessário avalia-los para obter evidências sobre os benefícios de sua adoção. O MEEGA+é um modelo para avaliar jogos educacionais usados no ensino de computação e analisa a experiência e percepção de aprendizagem do ponto de vista de alunos e instrutores. Apesar de fornecer modelos e planilhas predefinidas, não existe uma ferramenta de apoio que compreenda todo o processo de avaliação. Este artigo apresenta o Assistant MEEGA+, uma ferramenta web de apoio ao MEEGA+. Uma avaliação do Assistant MEEGA+ por instrutores e profissionais interessados no desenvolvimento e avaliação de jogos educacionais indicou resultados positivos em relação à utilidade, facilidade e intenção de usar a ferramenta. Os resultados encorajam a avaliação de jogos educacionais e a adoção da ferramenta. 
VII Congresso Brasileiro de Informática na Educação (CBIE 2018)

Anais do XXIX Simpósio Brasileiro de Informática na Educação (SBIE 2018)

\section{Introdução}

O uso de jogos educacionais tem se mostrado uma estratégia instrucional inovadora para o ensino de computação [Petri et al. 2017a]. Os jogos podem colocar os estudantes frente a situações reais do ambiente de trabalho, que, normalmente, são ausentes em atividades de sala de aula, como relacionamento em equipe, desenvolvimento de projetos e contato com stakeholders [Savi et al. 2011]. Frente à necessidade de oferecer oportunidades práticas para os estudantes, um grande número de jogos educacionais já foi desenvolvido [Battistella \& von Wangenheim, 2016].

Acredita-se que jogos educacionais forneçam muitos benefícios aos alunos, como o aumento do êxito da aprendizagem, do interesse e da motivação [Petri e von Wangenheim, 2018]. Apesar da motivação para o uso de jogos educacionais, são necessárias evidências sobre os benefícios do seu uso em sala de aula, justificando, assim, eventuais custos e esforços envolvidos ao optar pelo uso. Neste sentido, a avaliação de jogos educacionais torna-se necessária.

O modelo MEEGA destaca-se como modelo de avaliação de jogos educacionais por ser baseado em outros modelos de avaliação, na experiência do usuário e na taxonomia dos objetivos educacionais de Bloom [Savi et al. 2011]. Sua contribuição para a avaliação de jogos educacionais pode ser observada em Ferreira et al. (2014) e Araújo et al. (2017), entre outros trabalhos. Após realizar uma avaliação em larga escala sobre a confiabilidade e a validade dos constructos do MEEGA, constatou-se a necessidade de reformulação do modelo e reagrupamento de itens [Petri et al. 2017a]. Como resultado, foi proposto o MEEGA+ [Petri et al. 2017b] para avaliar jogos educacionais digitais e não digitais para ensino de Computação considerando a percepção da qualidade em termos de experiência do jogador e percepção da aprendizagem do ponto de vista de estudantes e instrutores. Como suporte, o MEEGA+ disponibiliza templates de questionários e planilhas para análise dos dados coletados. Contudo, não existe uma ferramenta de apoio que compreenda todas as fases do processo de avaliação de jogos educacionais usando o modelo MEEGA+.

O objetivo deste artigo é apresentar o Assistant MEEGA+, uma ferramenta desenvolvida para apoiar a adoção do modelo MEEGA+ para avaliação de jogos educacionais. O Assistant MEEGA+ é um sistema web, com layout responsivo, que contempla todas as etapas de aplicação do MEEGA+: planejamento e preparação do questionário, execução da avaliação com os usuários e sintetização de dados coletados. Através do processo de automatização e otimização das avaliações utilizando o modelo MEEGA+, espera-se encorajar a condução de avaliações de jogos educacionais e assim, apoiar uma maior utilização de jogos voltados para o processo de ensino-aprendizagem. Os resultados obtidos após uma avaliação inicial com dez instrutores e profissionais interessados no desenvolvimento e na avaliação de jogos educacionais indicam uma boa aceitação em relação à ferramenta, levando em consideração a percepção positiva em relação à facilidade de uso, utilidade e intenção de usar a ferramenta futuramente ao adotar ou desenvolver jogos educacionais.

O restante deste artigo está organizado da seguinte forma: a Seção 2 apresenta a fundamentação teórica e trabalhos relacionados sobre avaliação de jogos educacionais. A Seção 3 descreve o Assistant MEEGA+ e suas funcionalidades. Na Seção 4, a avaliação da ferramenta e os resultados são relatados. Por fim, na Seção 5, as considerações finais e trabalhos futuros são discutidos. 
VII Congresso Brasileiro de Informática na Educação (CBIE 2018)

Anais do XXIX Simpósio Brasileiro de Informática na Educação (SBIE 2018)

\section{Avaliação de Jogos Educacionais}

A fim de consolidar os benefícios do uso de jogos educacionais, é essencial avaliar esses jogos para obter evidências empíricas de sua qualidade como base para uma adoção efetiva e eficiente. E, assim, obter uma compreensão mais precisa dos resultados de seu uso, possibilitando identificar se os custos e esforço envolvidos em adotá-los são viáveis [Savi et al. 2011]. No entanto, para fornecer um apoio mais sistemático na avaliação de jogos, existem poucas tentativas para desenvolver abordagens para avaliar a qualidade de jogos educacionais [Petri e Gresse von Wangenheim, 2016]. Entre as abordagens para a avaliação de jogos encontrados na literatura, três se destacam: a escala EGameFLow [Fu et al., 2009], o framework proposto por Abdellatif et al. (2018) e o modelo MEEGA.

A escala EGameFlow [Fu et al. 2009], avalia jogos e-learning em termos de imersão, interação social, desafio, clareza de objetivos, feedback, concentração, controle e melhoria do conhecimento, utilizando um questionário para coleta de dados. A EGameFlow foi desenvolvida utilizando uma metodologia sistemática e é validada em termos de confiabilidade e validade por meio de quatro sessões de jogos. No entanto, embora a escala EGameFlow tenha apresentado validade e confiabilidade inicialmente satisfatórias, parece ter sido descontinuada por seus autores.

Abdellatif et al. (2018) propõem um framework que avalia a qualidade dos jogos em relação à usabilidade, engajamento, motivação, capacidade de compreensão e experiência do usuário. Este framework foi projetado para ser usado em estudos não experimentais. No entanto, ele não define explicitamente um instrumento de coleta de dados ou métodos de análise de dados. Além disso, o framework foi avaliado apenas parcialmente por meio de um estudo piloto, e nenhuma informação sobre sua validade e confiabilidade é relatada pelos autores.

O modelo MEEGA mede três fatores de jogos educacionais: motivação, experiência do usuário e aprendizagem. O MEEGA foi sistematicamente desenvolvido e define explicitamente o objetivo da avaliação e fornece um questionário padronizado para ser aplicado a fim de coletar dados sobre a percepção dos alunos usando um design de pesquisa não experimental (estudos de caso). O modelo também foi avaliado estatisticamente em termos de confiabilidade e validade com base em dados coletados de vários estudos de caso de diferentes autores. Atualmente, o modelo MEEGA parece ser usado mais amplamente na prática sendo relatado por vários estudos de autores diferentes, avaliando diferentes jogos e contextos [Calderón e Ruiz, 2015; Petri e Gresse von Wangenheim, 2016]. No entanto, os resultados de uma análise sistemática de uma avaliação em larga escala da versão inicial do modelo MEEGA, mostram que existem algumas limitações em termos de sua validade [Petri et al. 2017a], indicando uma sobreposição conceitual entre os fatores de motivação e usuário experiência. Com base na identificação destas oportunidades de melhoria, a versão inicial do modelo MEEGA é evoluída, desenvolvendo o modelo MEEGA+ [Petri et al. 2017b], que objetiva avaliar a qualidade de jogos educacionais para o ensino de computação, também por meio de realização de estudos de caso.

O MEEGA+ avalia a percepção da qualidade do jogo em termos da experiência do jogador e percepção da aprendizagem do ponto de vista de alunos e instrutores. $O$ fator de experiência do jogador é composto por um conjunto de dimensões: atenção focada, diversão, desafio, interação social, confiança, relevância, satisfação e 
VII Congresso Brasileiro de Informática na Educação (CBIE 2018)

Anais do XXIX Simpósio Brasileiro de Informática na Educação (SBIE 2018)

usabilidade. O fator de usabilidade está dividido em outras cinco subdimensões: aprendizibilidade, operabilidade, estética, acessibilidade e proteção de erro do usuário. O fator de aprendizagem percebida é subdividido em duas dimensões, a avaliação de curto prazo e o objetivo de aprendizagem. A avaliação de curto prazo objetiva avaliar o efeito global do jogo sobre a aprendizagem dos alunos. O objetivo de aprendizagem é personalizado, pois leva em consideração os objetivos de aprendizagem de cada jogo, tais como: análise, avaliação e criação [Petri et al. 2017b].

O modelo MEEGA+ fornece duas versões (jogo digital e jogo não-digital) de um questionário de avaliação que pode ser adaptado de acordo com os objetivos de aprendizagem do jogo educacional e uma planilha de análise dos dados. Para conduzir uma avaliação com o modelo MEEGA+ são necessárias algumas etapas: fazer adaptações no questionário, coletar os dados por meio dos questionários, tabular as respostas dos questionários utilizando a planilha de análise fornecida pelo modelo para que sejam gerados os gráficos com os resultados da avaliação.

\section{Assistant MEEGA+}

O Assistant MEEGA+ é uma ferramenta de apoio desenvolvida para plataforma web, que automatiza as etapas de avaliação de um jogo educacional com o modelo MEEGA+: planejamento, execução e análise de dados. O objetivo da ferramenta é melhorar a experiência dos usuários do MEEGA+, sejam eles os instrutores responsáveis pela avaliação, ou os alunos que respondem ao questionário.

Para os responsáveis pela avaliação, essa melhoria ocorre devido à mudança na maneira como os questionários são planejados e aplicados, assim como na forma como os dados coletados são analisados, retirando dos avaliadores a responsabilidade de tabular os dados dos questionários para a planilha de análise de dados, que acompanha $o$ MEEGA+. Vale destacar a redução de gastos com impressão, pois os alunos respondem aos questionários por meio de uma versão web responsiva da ferramenta na qual o questionário é apresentado e em que armazena as respostas dos alunos.

O Assistant MEEGA+ foi desenvolvido em PHP, com framework Codeigniter, AngularJS e banco de dados MySQL. Codeigniter é um framework PHP utilizado para desevolver o backend da ferramenta, pois oferece suporte otimizado para o roteamento e consistência do padrão MVC. Para o frontend foi utilizado o framework AngularJS, pois abstrai o acoplamento entre o client side e server side e traz característica do desenvolvimento backend para o lado do frontend, como o padrão MVC. O Assistant MEEGA+ possui duas versões: (a) versão que apoia o responsável pela avaliação, que está hospedada no subdomínio http://admin.assistantmeega.com.br/ como pode ser observada na Figura 1 (a); e (b) versão que apoia o aluno participante da avaliação, hospedada no domínio principal http://assistantmeega.com.br/, como pode ser observado na Figura 1 (b).

O questionário se assemelha ao modelo que hoje acompanha o MEEGA+. Algumas pequenas mudanças ocorreram, como o uso de avatares para representar cada opção de resposta, além de possuir navegação lateral entre os itens de avaliação do modelo MEEGA+ (perguntas). Após a condução da avaliação, os dados dos questionários respondidos podem ser acessados pelo responsável pela avaliação, que visualiza os gráficos de análise de dados propostos pelo modelo MEEGA+. 
VII Congresso Brasileiro de Informática na Educação (CBIE 2018)

Anais do XXIX Simpósio Brasileiro de Informática na Educação (SBIE 2018)

A

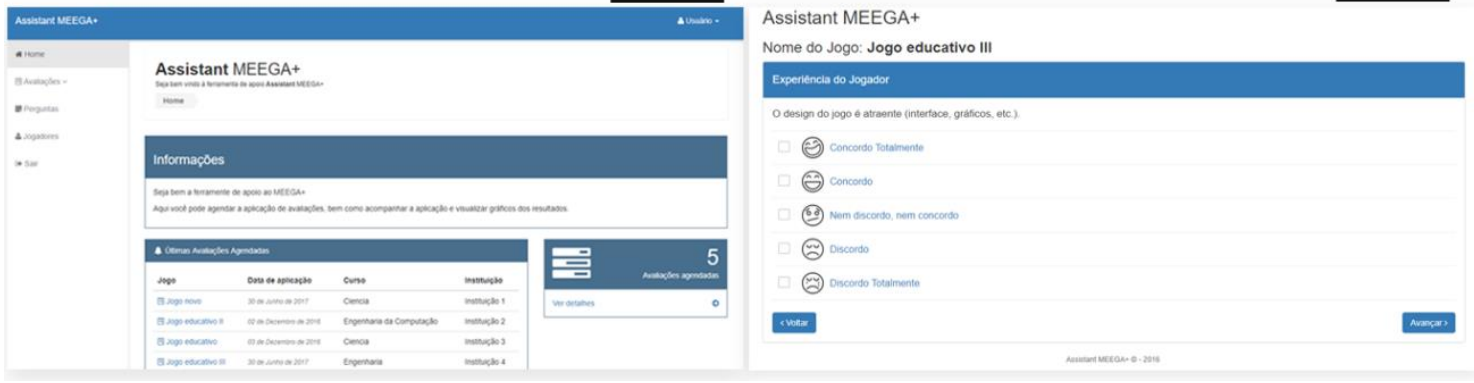

Figura 1. (A) Página inicial da Assistant MEEGA+; (B) Página para o aluno responder as perguntas do questionário.

\subsection{Planejamento de uma avaliação com o Assistant MEEGA+}

$\mathrm{Na}$ etapa de planejamento, o instrutor deve criar uma conta na ferramenta para então dar prosseguimento ao agendamento do planejamento da avaliação. A Figura 2 apresenta a página onde o instrutor pode planejar a avaliação e customizar o questionário.

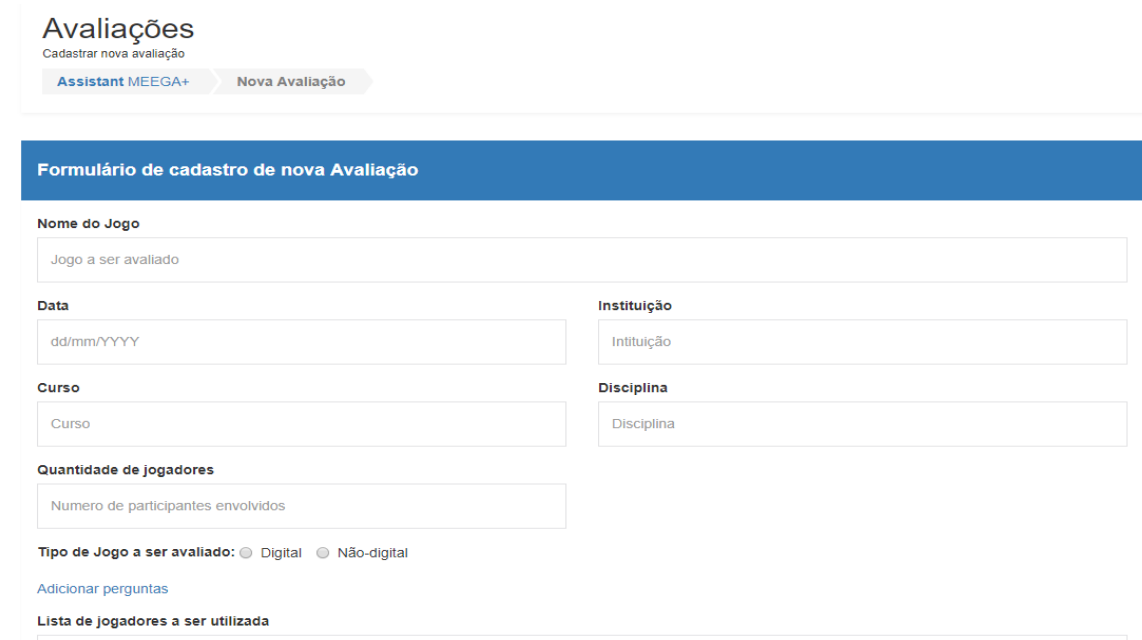

Figura 2. Página de planejamento de avaliação.

Nesta etapa, o instrutor deve preencher o formulário com informações básicas da avaliação do jogo. $\mathrm{O}$ cadastro de perguntas visa suprir uma característica importante do modelo MEEGA+, pois através dessas perguntas, os questionários se tornam únicos, assim, podendo adaptar-se ao objetivo especifico da avaliação.

\subsection{Condução de uma avaliação com o Assistant MEEGA+}

A aplicação do questionário é a etapa mais simples do uso da Assistant MEEGA+, pois o instrutor fica encarregado de disponibilizar o link personalizado gerado no momento do agendamento do questionário. Nessa etapa os alunos interagem com a ferramenta, através de um layout limpo e simples. A Figura 3 (a) apresenta a tela de coleta de dados demográficos, seguindo as diretrizes do modelo MEEGA+ e, a Figura 3 (b) apresenta a tela de coleta de respostas, contendo a categoria e o título da pergunta/afirmativa.

\subsection{Análise dos dados da avaliação com o Assistant MEEGA+}


VII Congresso Brasileiro de Informática na Educação (CBIE 2018)

Anais do XXIX Simpósio Brasileiro de Informática na Educação (SBIE 2018)

$\mathrm{Na}$ etapa de análise, o instrutor tem acesso, através da sua conta, aos gráficos gerados pela ferramenta, seguindo os padrões estabelecidos pelo documento de coleta de dados presente na aplicação do modelo MEEGA+.

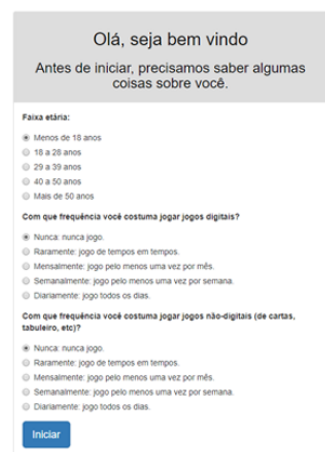

(a) Informações Demográficas

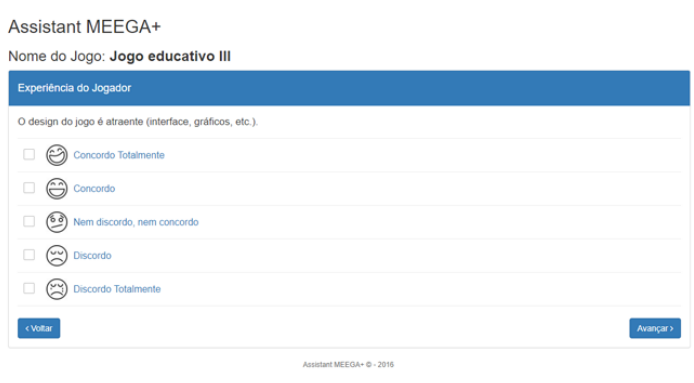

(b) Afirmação

Figura 3. Páginas da etapa de avaliação do jogo.

Na Figura 4 é apresentada a página de análise dos dados coletados em formato de gráficos, que seguem o padrão adotado pelo modelo MEEGA+. Além de visualizar e interagir com os gráficos de forma dinâmica, o instrutor também pode fazer o download dos mesmos.

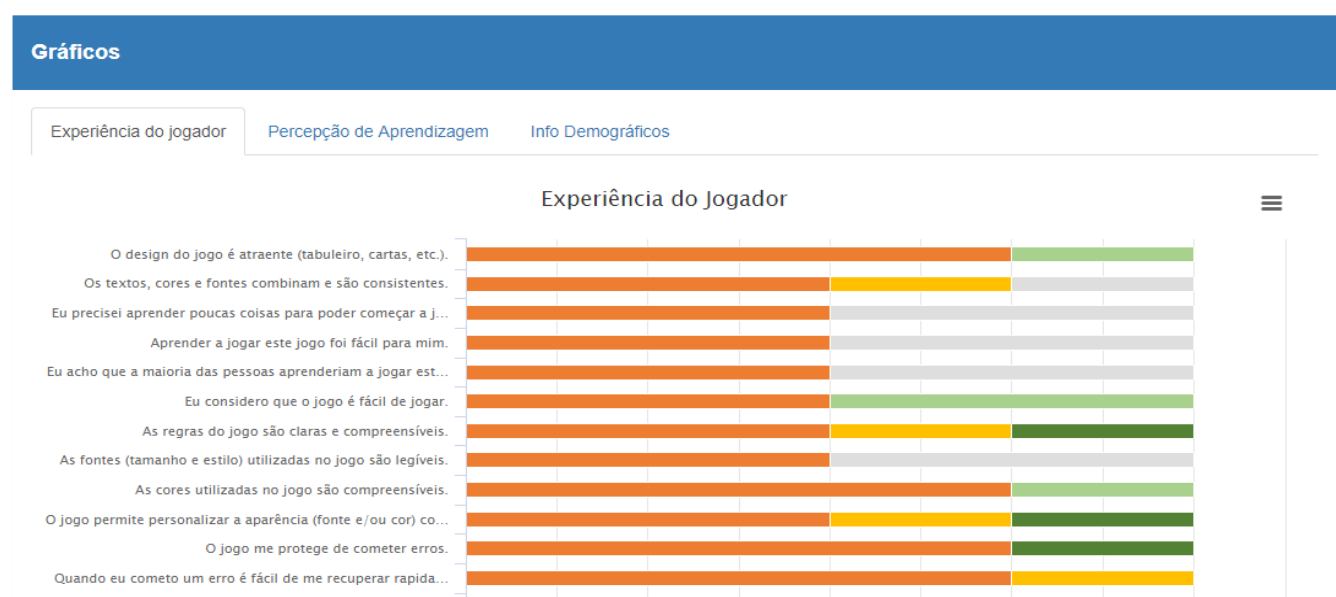

Figura 4. Página de análise de dados.

\section{Avaliação do Assistant MEEGA+}

Com o intuito de investigar se o Assistant MEEGA+ seria fácil de usar e se instrutores e profissionais teriam intenção em adotá-lo, foi conduzido um teste com usuários. Para mensurar a aceitação da ferramenta, foram adotados três indicadores do Modelo de Aceitação de Tecnologia (TAM 3): utilidade percebida, facilidade de uso percebida e intenção de uso [Vekantesh \& Bala, 2008]. O modelo TAM é amplamente utilizado para avaliar a aceitação de novas tecnologias e permite mensurar a percepção dos usuários por meio de um conjunto de afirmativas sobre cada indicador e uma escala de Likert por meio do qual o usuário fornece sua concordância em relação às afirmativas [Conte et al. 2018].

\subsection{Planejamento e execução}


VII Congresso Brasileiro de Informática na Educação (CBIE 2018)

Anais do XXIX Simpósio Brasileiro de Informática na Educação (SBIE 2018)

A avaliação do Assistant MEEGA+ foi conduzida em ambiente acadêmico. Os usuários foram dez profissionais interessados no desenvolvimento e avaliação de jogos educacionais com um dos seguintes perfis: (a) sete instrutores de cursos de computação e (b) três pós-graduandos em Engenharia de Software com interesse no desenvolvimento de jogos educacionais. Todos os usuários que participaram da avaliação já haviam utilizado o modelo MEEGA+ previamente na avaliação de jogos educacionais, como instrutor ou como aluno. As tarefas a serem realizadas pelos usuários no Assistant MEEGA+ estavam relacionadas ao planejamento de uma avaliação com o modelo MEEGA+: (i) criar nova conta de instrutor; (ii) cadastrar novas perguntas; e (iii) planejar nova avaliação. Um roteiro de tarefas foi elaborado para que fosse seguido durante a avaliação pelos usuários.

As métricas utilizadas para avaliar a aceitação do Assistant MEEGA+ foram: (i) utilidade percebida, que define o grau no qual uma pessoa considera que usar uma tecnologia melhoraria seu desempenho em determinadas atividades; (ii) facilidade de uso percebida, que define o grau no qual uma pessoa considera que usar uma tecnologia seria livre de esforço; e (iii) intenção de uso, que define o grau no qual uma pessoa prevê que utilizaria uma tecnologia no futuro [Vekantesh \& Bala, 2008]. Um questionário foi elaborado contendo as afirmativas apresentadas e um espaço para comentários adicionais sobre o Assistant MEEGA+.

A execução da avaliação do Assistant MEEGA+ ocorreu ao longo de uma semana. Cada usuário recebia uma breve explicação sobre o objetivo da ferramenta, suas principais funcionalidades e em seguida, recebia o roteiro de tarefas a ser seguido durante o teste. Todos os usuários tiveram acesso à ferramenta através do endereço http://assistantmeega.com.br/ e assim puderam realizar os passos propostos no roteiro de tarefas. Não foi necessário em nenhum momento o auxílio externo. Após a realização das tarefas, os usuários responderam ao questionário contendo as afirmativas adaptadas do modelo TAM 3. No mesmo questionário, os usuários também poderiam fornecer comentários adicionais sobre a ferramenta.

\subsection{Análise dos Resultados}

A Figura 5 apresenta os resultados observados para o indicador de utilidade percebida do modelo TAM. Observa-se que seis usuários concordam totalmente com todas as afirmativas relacionadas à utilidade da ferramenta. Outros quatro usuários concordaram amplamente ou parcialmente com as mesmas afirmativas.

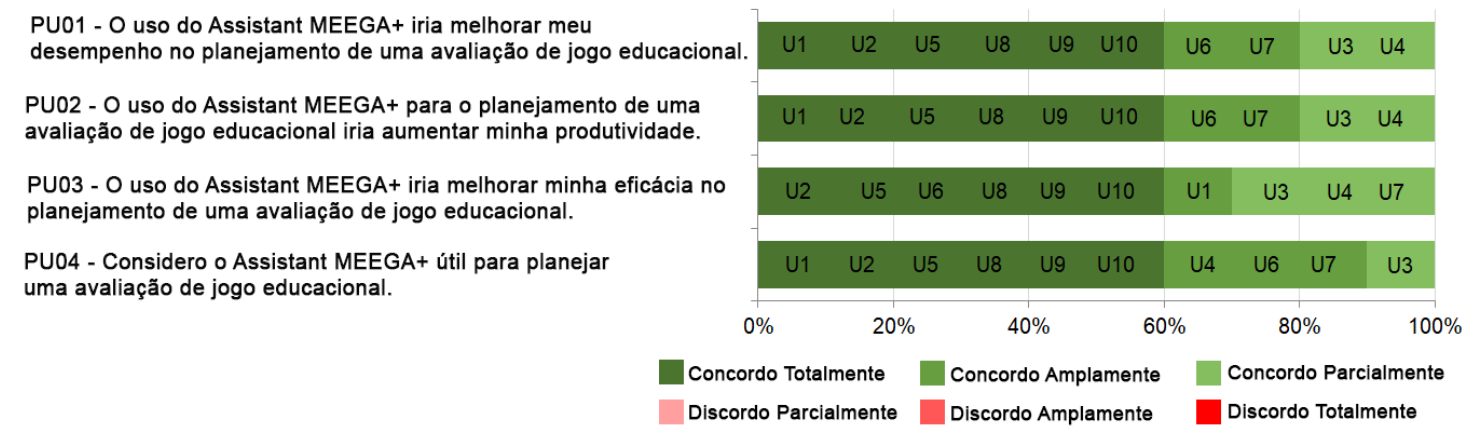

Figura 5. Avaliação da utilidade percebida pelos usuários.

Não foram observadas indicações de discordâncias em relação às afirmativas que mensuram este indicador. Apesar dos resultados, em geral, positivos, nota-se que 
VII Congresso Brasileiro de Informática na Educação (CBIE 2018)

Anais do XXIX Simpósio Brasileiro de Informática na Educação (SBIE 2018)

três usuários concordaram parcialmente que o Assistant MEEGA + melhoraria sua eficácia no planejamento de avaliações (PU03).

Na Figura 6, observam-se os resultados obtidos em relação às afirmativas que mensuram o indicador de facilidade de uso percebida. Apesar de a maioria dos usuários concordarem (totalmente ou não) com todas as afirmativas, foram observadas respostas negativas em relação à clareza e à facilidade de entendimento das funcionalidades (PEOU1), necessidade de muito esforço mental para usar a ferramenta (PEOU2) e facilidade de uso da ferramenta (PEOU3). Ao analisar os comentários adicionais dos usuários, notou-se que as dificuldades estavam relacionadas ao preenchimento de dados, realização de cadastro e navegação da ferramenta.

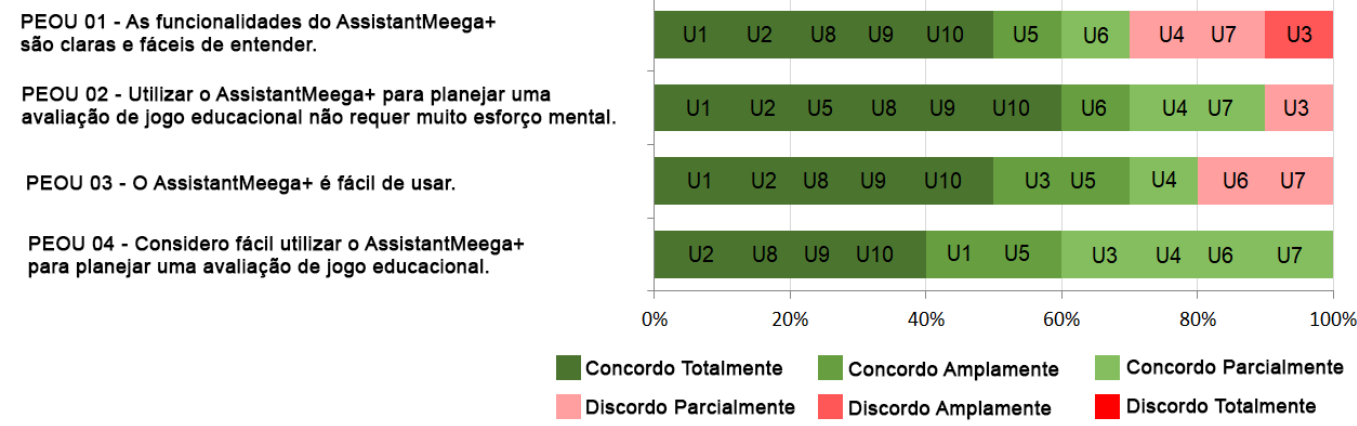

Figura 6. Avaliação da facilidade de uso percebida pelos usuários.

Alguns comentários dos usuários auxiliaram a entender melhor os aspectos negativos da facilidade de uso da ferramenta, como podem ser observados logo abaixo.

“A aplicação deveria apresentar suporte para os usuários. Por exemplo: algum texto de ajuda nos campos a serem preenchidos." - U5

"A ferramenta tem alguns problemas de navegação, icones ocultos, opções dificeis de encontrar. Alguém leigo pode se perder na ferramenta. Será que poderia ter um tutorial inicial ou ajuda dos passos para usar a ferramenta?" - U10

"Os formulários/questionários/perguntas devem ter a identificação dos campos obrigatórios"'- U7

"Não consegui me cadastrar com facilidade no sistema. Creio que um usuário leigo terá dificuldades em utilizar o mesmo." - U3

A Figura 7 ilustra os resultados obtidos para o indicador de intenção de uso. Observa-se que seis usuários concordaram totalmente com as afirmativas que mensuram a intenção de uso. Analisando os comentários de alguns usuários, notou-se que a possibilidade de gerar automaticamente os gráficos sem a necessidade da etapa de tabulação de dados manual impactou positivamente na aceitação da ferramenta

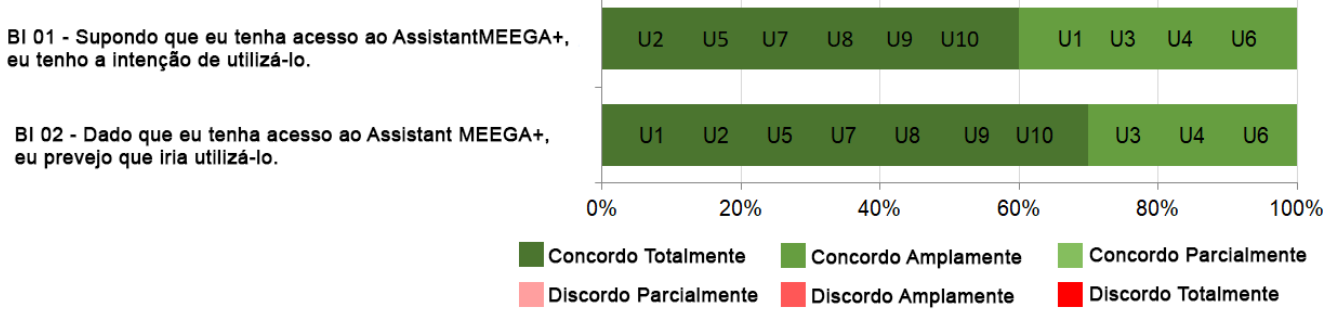

Figura 7. Avaliação da intenção de usar o Assistant MEEGA+. 
VII Congresso Brasileiro de Informática na Educação (CBIE 2018)

Anais do XXIX Simpósio Brasileiro de Informática na Educação (SBIE 2018)

"A ferramenta auxilia no auxílio do planejamento da avaliação de jogos educacionais, uma vez que isto era feito a partir da planilha e dava mais trabalho." U6

"Acredito que fica mais fácil gerar os gráficos, uma vez que os dados já serão todos tabulados pela ferramenta." - U5

Os resultados indicam que apesar dos resultados positivos na avaliação dos aspectos de utilidade percebida e intenção de uso, algumas características do Assistant MEEGA+ podem ser reprojetadas, visando uma maior facilidade de uso. Como possíveis soluções, um tutorial de uso pode ser apresentado no primeiro acesso de um usuário, descrições e dicas sobre campos e funcionalidades da ferramenta podem ser fornecidas. Além disso, a navegação e os ícones utilizados na ferramenta podem ser repensados por meio da adoção de técnicas de Interação Humano-Computador, como Card Sorting.

\section{Considerações Finais e Trabalhos Futuros}

Este artigo apresentou o Assistant MEEGA+, uma ferramenta de apoio ao processo de avaliação de jogos educacionais com o modelo MEEGA+. A ferramenta engloba as três etapas essenciais na aplicação do modelo MEEGA+: planejamento, condução e análise de dados. Espera-se que com a adoção do Assistant MEEGA+, os instrutores responsáveis pela avaliação possam otimizar o esforço para planejamento, condução e análise dos resultados de uma avaliação. Os riscos relacionados aos erros na tabulação dos dados coletados também podem ser reduzidos e resultados consistentes podem ser gerados, uma vez que a geração dos gráficos é automática. Espera-se também reduzir o custo com a preparação dos materiais de coleta de dados, pois a ferramenta dispensa a necessidade de materiais impressos para a avaliação e elimina a etapa de tabulação dos dados em planilha para a obtenção dos resultados. E com a automatização e otimização das avaliações utilizando o modelo MEEGA+, espera-se encorajar a condução de avaliações de jogos educacionais e assim, apoiar uma maior utilização de jogos educacionais.

Os resultados da avaliação da ferramenta foram positivos no que diz respeito à utilidade percebida e intenção dos usuários de usar a ferramenta futuramente. Os usuários indicaram a geração automática dos gráficos de análise de dados sem a necessidade da tabulação manual dos dados como justificativa para o uso futuro do Assistant MEEGA+. Todavia, foram identificadas necessidades de melhorias referentes à facilidade de uso da ferramenta, tais como dificuldades no cadastro de novo usuário, pouca informação sobre os campos para preenchimento de dados e dificuldades na navegação da ferramenta.

Como trabalhos futuros, é possível implementar as melhorias referentes à facilidade de uso da ferramenta, como (i) tutorial de uso, (ii) informações e dicas sobre os preenchimentos de dados de entrada e (iii) reprojeto da navegação da ferramenta. Além destes pontos, também é possível implementar suporte a outros idiomas, como inglês e espanhol, levando em consideração o suporte fornecido pelo modelo MEEGA+ atualmente. Por fim, como este artigo focou na avaliação das funcionalidades de planejamento, novos testes devem ser conduzidos com foco nas funcionalidades de execução e análise dos dados de uma avaliação de jogos educacionais. 
VII Congresso Brasileiro de Informática na Educação (CBIE 2018)

Anais do XXIX Simpósio Brasileiro de Informática na Educação (SBIE 2018)

\section{Agradecimentos}

Os autores agradecem ao apoio financeiro fornecido a esta pesquisa pela CAPES processo 175956/2013 e CNPq processos 423149/2016-4, 311494/2017-0 e 302149/2016-3.

\section{Referências}

Abdellatif, A. J., McCollum, B., \& McMullan, P. (2018). Serious Games: Quality Characteristics Evaluation Framework and Case Study. Proc. of IEEE Integrated STEM Education Conference, pp. 112-119.

Araujo, N., Machado, R., Viana, D., \& Rivero, L. (2017). Avaliando a Viabilidade do BlackBox em Sala de Aula: Um Jogo Sério para Ensino de Teste Funcional de Software. Anais do XXVIII Simpósio Brasileiro de Informática na Educação (SBIE), pp. 817-826.

Battistella, P., \& von Wangenheim, C. G. (2016). Games for teaching computing in higher education-a systematic review. IEEE Technology and Engineering Education Journal, 9(1), pp. 8-30.

Calderón, A. \& Ruiz M. (2015). A systematic literature review on serious games evaluation: An application to software project management. Computers \& Education, 87, pp. 396-422.

Conte, T., Valentim, N., Cabrejos, L.J., Lopes, A., Oliveira, E., Steinmacher, I. (2018) Modelo de Aceitação de Tecnologia. Automatização de Teste de Software com Ferramentas de Software Livre. 1ed.: Elsevier Editora Ltda., 2018, v. 1, pp. 229-238.

Ferreira, B. M., Rivero, L., Lopes, A., Marques, A. B., \& Conte, T. (2014). UsabiliCity: um jogo de apoio ao ensino de propriedades de usabilidade de software através de analogias. Anais do XXV Simpósio Brasileiro de Informática na Educação (SBIE), pp. 1273-182.

Petri, G., Gresse von Wangenheim, C., \& Borgatto, A. F. (2017a). A large-scale evaluation of a model for the evaluation of games for teaching software engineering. In Proc. of International Conference on Software Engineering: Software Engineering Education and Training Track (ICSE-SEET), pp. 180-189.

Petri, G., Gresse von Wangenheim, C., \& Borgatto, A. F. (2017b). Evolução de um Modelo de Avaliação de Jogos para o Ensino de Computação. Anais do XXV Workshop sobre Educação em Computação (WEI), pp.2327-2336.

Petri, G. e Gresse von Wangenheim, C. (2016). How to Evaluate Educational Games: a Systematic Literature Review. Journal of Universal Computer Science, 22(7), pp. 992-1021.

Savi, R., Gresse von Wangenheim, C., e Borgatto, A. (2011). Um Modelo de Avaliação de Jogos Educacionais na Engenharia de Software. Anais do XXV Simpósio Brasileiro de Engenharia de Software (SBES 2011), pp. 194-203.

Venkatesh, V., \& Bala, H. (2008). Technology acceptance model 3 and a research agenda on interventions. Decision sciences, 39(2), pp. 273-315. 